\title{
Postoperative Complications and Reoperation Rates Following Open Reduction and Internal Fixation of Ankle Fracture
}

\author{
Armando Macera ${ }^{1}$ Christian Carulli ${ }^{2}$ Luigi Sirleo ${ }^{2}$ Massimo Innocenti ${ }^{2}$ \\ ${ }^{1}$ Hospital Universitario Infanta Elena, Madrid, Spain \\ 2 Orthopaedic Clinic, University of Florence, Florence, Italy \\ Address for correspondence Armando Macera, MD, Hospital \\ Universitario Infanta Elena, Avenida Reyes Catolicos 21, 28340 \\ Valdemoro, Madrid, Spain (e-mail: armandomacera@hotmail.it). \\ Joints 2018;6:110-115.
}

\begin{abstract}
Purpose The purpose of this study was to determinate the overall postoperative complication and reoperation rates related to open reduction and internal fixation (ORIF) of ankle fractures.

Methods All patients who had undergone an ankle fracture operation at our institution from January 2005 through December 2013 were identified by querying the hospital surgical procedure database for diagnoses codes. Medical records, surgical procedure, and outpatient control reports were reviewed to collect pre-, intra-, and postoperative details. All data obtained were retrospectively analyzed by the authors to evaluate the postoperative complications and the type of further surgical treatment required to treat them.

Results A total of 378 consecutive patients were included in the study. Overall complications rate was $36.0 \%$. Minor complications (4.5\%) were represented by superficial infection (1.3\%) and impaired wound healing (3.2\%). All these patients required advanced wound care and prolonged oral antibiotics. Major complications (31.5\%) included: residual pain (17.2\%), deep infection (3.4\%), malunion (2.4\%), posttraumatic ankle osteoarthritis (5.0\%), implant breakage (0.3\%), complex regional pain syndrome (1.3\%), and arthrofibrosis $(1.9 \%)$. Note that $21.7 \%$ of major complications required further surgical procedure. Reoperations included arthroscopic debridement (15.1\%), hardware removal and debridement of all necrotic tissue (4.5\%), and

\section{Keywords}

- ankle fracture

- open reduction and internal fixation postoperative

- complication

- reoperation ankle fusion (2.1\%). Surgery was necessary mainly for pain removal and function recovery.

Conclusion Ankle fracture ORIF represents a satisfying surgical treatment. Nevertheless, postoperative complications are not uncommon. Minor complications can be easily managed with medications and repeated outpatient controls. Reoperation is occasionally required to treat major complications. Revision surgery is mandatory to ensure pain relief and function improvement.

Level of Evidence Level II, retrospective cohort study.
\end{abstract}

received

September 1, 2017

accepted after revision

April 8, 2018

published online

May 21, 2018
DOI https://doi.org/

$10.1055 / \mathrm{s}-0038-1653949$.

ISSN 2282-4324.
Copyright @ 2018 Georg Thieme Verlag License terms

KG Stuttgart · New York
(๑) $\Theta \circledast$ 


\section{Introduction}

Surgical treatment of ankle fracture is one of the most commonly performed orthopaedic procedures. ${ }^{1,2}$ Furthermore, ankle fracture incidence have increased significantly in the last decades and young patients and workers are often involved. $^{2-4}$ Open reduction and internal fixation (ORIF) represents the gold standard for the treatment of ankle fractures to restore anatomical alignment and articular congruity of ankle mortise to avoid altered loading of the tibiotalar joint and subsequent poor functional outcomes. ${ }^{5,6}$ Although results are generally favorable, postoperative complications are not uncommon and have a considerable impact on postoperative morbidity, life quality worsening, and health care costs. ${ }^{7-9}$ Despite that, the literature is poor of studies that report the overall complication rates following ORIF of ankle fractures, rates varying from 1 to $40 \%{ }^{3,10,11}$ The purpose of this study was to determinate the overall postoperative complication and reoperation rates related to ORIF of ankle fractures.

\section{Methods}

\section{Study Design}

This study was designed as a retrospective cohort study. Medical records, surgical procedure, and outpatient control reports were reviewed to collect pre-, intra-, and postoperative details. All data obtained were retrospectively analyzed to evaluate the postoperative complications and the type of further surgical treatment required to treat them.

The study fulfills the criteria of the Declaration of Helsinki and has been approved by the Institutional Review Board of our institution.

\section{Patient Selection}

All patients who had undergone an ankle fracture operation at our institution from January 2005 through December 2013 were identified by querying the hospital surgical procedure database for diagnoses codes with International Classification of Diseases - 9th revision -Clinical Modification (ICD-9$\mathrm{CM})^{12}$ for ankle fracture: medial malleolar fracture, closed (824.0) and open (824.1); lateral malleolar fracture, closed (824.2) and open (824.3); bimalleolar fracture, closed (824.4) and open (824.5); trimalleolar fracture, closed (824.6) and open (824.7); and unspecified ankle fracture, closed (824.8) and open (824.9). Diagnoses codes with ICD-9-CM for closed ankle dislocation (837.0) and open ankle dislocation (837.1) were also searched for ankle fracture identification.

Inclusion criteria were: patients 18 years of age or older, unilateral, isolated closed or open ankle fracture/ankle fracture dislocation, and all patients had to be definitively treated with ORIF, with minimum 12-month outpatient follow-up. Exclusion criteria were: associated fractures of the fibula, tibia, or talus and polytrauma.

\section{Treatment Protocol}

A standardized operative and postoperative protocol was used at our institution during the period under investigation.
Preoperative and 24-hour postoperative prophylactic intravenous antibiotics, as specified in the hospital formulary, were used in all cases. Generally, a spinal anesthesia was conducted; only in case of contraindication to the use of local anesthetic, a general anesthesia was performed. All operations were performed using a pneumatic ischemia under tourniquet control. All fractures were treated using the same approach and surgical technique based on Arbeitsgemeinschaft für Osteosynthesefragen (AO) principles. ${ }^{13}$ Fluoroscopic intensifier was used to perform the surgical procedure and to evaluate the fracture reduction and the stability of fixation in all cases. Wound was closed using the same three-layer method (peroneal fascia, subcutaneous tissue, skin).

A standard postoperative care protocol was followed as a rule. During first 2 weeks after surgery, a posterior short leg splint with the foot at $90^{\circ}$ was applied and no weight-bearing was permitted to improve wound healing. Two weeks postoperatively, sutures were removed. According to fracture patterns and wound state, a rehabilitation program with passive and active range of motion (ROM) exercises and partial weight-bearing of up to 10 to $15 \mathrm{~kg}$ was allowed to favor fracture healing. Full weight-bearing and full normal activity was authorized once the bone and ligamentous healing was assured (6 weeks postoperatively), depending on the fracture pattern and follow-up X-ray findings.

In case of syndesmotic tear, no weight-bearing was allowed until the removal of the positioning screw; the latter was performed after 8 weeks in young and active patients and after 12 weeks in very unstable fractures, diabetic patients, and smokers. Full weight-bearing and full normal activity were allowed after screw removal.

\section{Clinical Evaluation}

Medical records were reviewed to identify baseline characteristics including age, gender, body mass index, type of injury (closed or open), delay to surgery, and days to discharge.

Outpatient control reports were analyzed to collect postoperative data including: functional outcome measurement, clinical fracture healing, residual pain, ROM, and wound inspection.

Functional outcome measurement and clinical fracture healing for the management of complications were evaluated at 6-month outpatient follow-up using the American Orthopaedic Foot and Ankle Society Ankle-Hindfoot Scale (AOFASAHS $)^{14}$ and the Olerud-Molander Ankle Score (OMAS). ${ }^{15}$ AOFAS-AHS covers three categories: pain, function, and alignment. OMAS scale is based on nine different items: pain, stiffness, swelling, stair climbing, running, jumping, squatting, supports, and works/activities of daily living. Both score systems have result ranging from 0 (totally impaired) to 100 (completely unimpaired).

Residual pain was assessed using the subjective Visual Analogue Scale pain scale with result ranging from 0 (no pain) to 10 (worst pain). Total ROM of the ankle joint was measured using a goniometer to identify limitation. Wound inspection was performed within 4 weeks postoperatively to identify wound closure problems or surgical site infection (SSI) and results were recorded by applying the criteria of the 
112 Ankle Fracture ORIF: Postoperative Complications and Reoperation Rates Macera et al.

Center for Disease Control and Prevention. ${ }^{16}$ SSIs were classified into superficial and deep infection.

\section{Radiological Evaluation}

Radiological evaluation was performed on anteroposterior, mortise, and lateral view. Preoperative X-rays were observed to describe ankle fracture patterns following anatomic/ descriptive classification. The type of fracture was identified according to the Danis-Weber criteria. ${ }^{17}$ Radiographic criteria ${ }^{15}$ were applied on postoperative X-rays including: ankle joint congruency, radiological fracture healing, and osteoarthritis $(\mathrm{OA})$ grade. Several radiographic grading systems have been developed for the ankle OA evaluation. ${ }^{18}$ Moon et $\mathrm{al}^{19}$ compared the van Dijk scale, ${ }^{20}$ the modified Kellgren-Lawrence scale, ${ }^{21}$ and modified Takakura scale, ${ }^{22}$ and concluded that all these scales were reliable and valid. ${ }^{18}$ In clinical practice, patients with Kellgren-Lawrence 1, 2, or 3 and van Dijk 1 or 2 scales are diagnosed in an early-stage ankle OA and those with Kellgren-Lawrence 3 or 4 and van Dijk 3 scales in an advanced-stage ankle $\mathrm{OA}^{23}$

\section{Complications}

Clinical and radiographic outcomes were observed during 24-month outpatient follow-up after ankle fractures. Postoperative complications and further surgical treatment required after ORIF ankle fracture were recorded.

Superficial infection and impaired wound healing were considered minor complications. Deep infection, residual pain, OA, malunion, arthrofibrosis, complex regional pain syndrome (CRPS), and implant breakage were considered major complications.

\section{Results}

We identified 1,112 consecutive closed and open ankle fractures/fracture dislocations that were diagnosed at our institution between January 2005 and December 2013. Out of 426 fractures that were definitively treated with ORIF, 36 were excluded before surgery because they mismatched the inclusion criteria, 4 were excluded during surgery for iatrogenic fracture or intraoperative associated fracture identifications, and 8 were excluded after surgery for unplanned or independent hardware removal. Overall, 378 patients (378 fractures) were included in the study. Of these, 264 patients were males (69.8\%) and 114 females (30.2\%) and the mean age was 47.2 years. A mean of 2.7 days from injury to surgery and a mean of 2.2 days to discharge after surgical treatment were recorded. Baseline characteristics of the included patients are reported in -Table 1. The number of treating surgeons was 21, including residents and orthopaedic surgeons.

Fracture patterns are shown in -Table 2. Bimalleolar fractures (45.8\%) were the most frequent ankle fracture followed by isolated medial and isolated lateral fractures (19.6 and $15.9 \%$, respectively). Twenty-two cases (5.8\%) were ankle fracture dislocations and 20 cases (5.3\%) were open ankle fractures. According to the Danis-Weber criteria, ${ }^{17} 110$ (29.1\%) A-type fractures, 216 (57.1\%) B-type fractures, and 52 (16.8\%) C-type fractures were identified (-Table 3).
Table 1 Baseline characteristics of included patients ${ }^{a}$

\begin{tabular}{|l|l|}
\hline Age (y) & $47.2(18-64)$ \\
\hline Gender $^{\mathrm{b}}$ & \\
\hline Male & $264(69.8 \%)$ \\
\hline Female & $114(30.2 \%)$ \\
\hline BMI & $22.6(18.5-35.4)$ \\
\hline Time from injury to surgery (d) & $2.7(0-13)$ \\
\hline Discharge time (d) & $2.2(1-4)$ \\
\hline
\end{tabular}

Abbreviation: BMI, body mass index.

${ }^{a}$ Values are expressed as mean (range) unless otherwise specified.

${ }^{b}$ Values are expressed as number of patients and (percentage).

Table 2 Fracture patterns ${ }^{\mathrm{a}}$ (anatomic/descriptive classification)

\begin{tabular}{|l|l|l|}
\hline & Closed fracture & Open fracture \\
\hline Isolated lateral & $60(15.9 \%)$ & - \\
\hline Isolated medial & $74(19.6 \%)$ & - \\
\hline Bimalleolar & $157(41.5 \%)$ & $16(4.3 \%)$ \\
\hline Trimalleolar & $47(12.4 \%)$ & $2(0.5 \%)$ \\
\hline Fracture dislocation & $20(5.3 \%)$ & $2(0.5 \%)$ \\
\hline Total & $358(94.7 \%)$ & $20(5.3 \%)$ \\
\hline
\end{tabular}

${ }^{a}$ Values are expressed as number of fractures and (percentage).

Table 3 Type of fractures ${ }^{a}$ (Danis-Weber classification)

\begin{tabular}{|l|l|}
\hline A-type (infrasyndesmotic) & $110(29.1 \%)$ \\
\hline B-type (transsyndesmotic) & $216(57.1 \%)$ \\
\hline C-type (suprasyndesmotic) & $52(13.8 \%)$ \\
\hline Total & 378 \\
\hline
\end{tabular}

${ }^{a}$ Values are expressed as number of fractures and percentage.

All fractures healed at the expected time. The mean AOFAS-AHS and OMAS scores after surgery at 6-month follow-up were 83.2 (range, 80-96) and 89 (range, 76$100)$, respectively. Outcomes were negatively influenced by complications. Particularly, worse categories were represented by pain in the majority of patients (115 subjects), stiffness in 27 patients, and swelling in 50 patients.

Complications were recorded in 136 cases (36\%) (-Table 4). Minor complications were recorded in 17 patients (4.5\%), superficial infection in 5 patients (1.3\%), and impaired wound healing (dehiscence, edge necrosis, blistering) in 12 patients (3.2\%). All these patients required advanced wound care and prolonged oral antibiotics performed in outpatient controls.

Major complications were found in 119 patients (31.5\%): residual pain in 65 patients (17.2\%), deep infection in 13 patients (3.4\%), malunion in 9 patients (2.4\%), advanced posttraumatic ankle OA in 19 patients (5.0\%), implant breakage in 1 patient (0.3\%), CRPS in 5 patients (1.3\%), and arthrofibrosis in 7 patients (1.9\%). According to the van Dijk $^{20}$ and modified Kellgren-Lawrence criteria, ${ }^{21}$ advanced 
Table 4 Postoperative complication rates ${ }^{\mathrm{a}}$

\begin{tabular}{|l|l|}
\hline Major complications & \\
\hline Residual pain & $65(17.2 \%)$ \\
\hline Advanced posttraumatic OA & $19(5.0 \%)$ \\
\hline Deep infection & $13(3.4 \%)$ \\
\hline Malunion & $9(2.4 \%)$ \\
\hline Arthrofibrosis & $7(1.9 \%)$ \\
\hline Complex regional pain syndrome & $5(1.3 \%)$ \\
\hline Implant breakage & $1(0.3 \%)$ \\
\hline Minor complications & \\
\hline Superficial infection & $5(1.3 \%)$ \\
\hline Impaired wound healing & $12(3.2 \%)$ \\
\hline Total & $136(36.0 \%)$ \\
\hline
\end{tabular}

Abbreviation: OA, osteoarthritis.

${ }^{a}$ Values are expressed as number of complications and percentage.

Table 5 Advanced posttraumatic OA related to fracture type ${ }^{a}$ (Danis-Weber classification)

\begin{tabular}{|l|l|}
\hline Fractures & Advanced OA \\
\hline A-type (infrasyndesmotic) & $0 / 110(0.0 \%)$ \\
\hline B-type (transsyndesmotic) & $12 / 216(5.5 \%)$ \\
\hline C-type (suprasyndesmotic) & $7 / 52(13.5 \%)$ \\
\hline Total & $19 / 378(5.0 \%)$ \\
\hline
\end{tabular}

Abbreviation: OA, osteoarthritis.

${ }^{a}$ Values are expressed as number of fractures and percentage.

posttraumatic ankle OA were identified in 12 (5.5\%) B-type fractures and in 7 (13.5\%) C-type fractures (-Table 5).

Eighty-two patients (21.7\%) affected by major complications required further surgical procedure. - Table 6 shows reoperation rates related to fracture type according to the Danis-Weber classification. ${ }^{17}$ Open debridement of all necrotic and fibrous tissue and hardware removal was indicated in 17 cases (4.5\%) for malunions, infections, extra-articular impingement, and implant breakage; arthroscopic debridement was necessary in 57 cases $(15.1 \%)$ for residual pain, intra-articular impingement, arthrofibrosis, and early posttraumatic ankle $\mathrm{OA}$; and ankle fusion was needed in 8 cases (2.1\%) for advanced posttraumatic ankle OA. Surgery was necessary mainly for pain removal and function recovery. During the study, eight patients underwent unplanned or independent hardware removal. No complications were recorded in these cases, but they were excluded from the study.

\section{Discussion}

Overall complication rates following ORIF of ankle fractures widely vary in the literature, ranging from 1 to $40 \%$. $3,10,11$ These data are comparable to $36.0 \%$ of overall complication rate reported in this study. Residual pain, SSI, advanced posttraumatic ankle $\mathrm{OA}$, and wound dehiscence are common postoperative complications of ankle fracture, as reported in different studies. ${ }^{1,8,11,24-31}$

Postoperative wound infection is one of the most common complications of ankle fracture surgery. ${ }^{25}$ Overall SSI rate reported in the literature varies from 1.4 to $13.0 \%{ }^{6,27,28} \mathrm{In}$ detail, prevalence ranges between 3.0 and $10.0 \%$ in case of superficial infection ${ }^{9,27}$ and between 1.0 and $6.8 \%^{6,9,27}$ in case of deep infection. These reported values are slightly higher than those reported in this study (4.7\% for overall SSIs, 1.3 and $3.4 \%$ for superficial and deep infection, respectively).

Goost et $\mathrm{al}^{32}$ and Lindsjö ${ }^{33}$ reported an advanced posttraumatic ankle $\mathrm{OA}$ in 10.0 and $14.0 \%$ of the cases, respectively. These rates were higher than that reported in this study. Lübbeke et $\mathrm{al}^{34}$ reported an advanced ankle $\mathrm{OA}$ at 18 -year follow-up in 53 and 31\% of Weber-C and Weber-B cases, respectively. Fleischer and Warncke ${ }^{35}$ reported $\mathrm{OA}$ 8 years after ankle fracture osteosyntheses in 46 and 28\% of Weber-C and Weber-B fractures, respectively. Lindsjö ${ }^{33}$ reported advanced OA 2- to 6 years after ankle fracture dislocations in 33 and $12 \%$ of Weber- $\mathrm{C}$ and Weber-B fractures, respectively. Müller et a ${ }^{36}$ reported an advanced $O A$ in $38 \%$ of Weber- $C$ fractures and in $14 \%$ of Weber-B fractures at 4 to 12 years postoperatively. These reported prevalence data are higher than that observed in this study (13.5 and 5.5\% in Weber-C and Weber-B fractures, respectively). Discrepancy might be explained with duration of follow-up.

In our series, pure residual pain after ankle fracture ORIF was found in $17.2 \%$ of patients. Postoperative ankle residual pain is also frequently related to chondral injuries and softtissue impingement, ${ }^{7,26,37-39}$ as well as posttraumatic neuromas, ${ }^{40}$ arthrofibrosis, ${ }^{41}$ malreduction, loss of reduction, and malunion. ${ }^{6,42,43}$

Table 6 Reoperation rates related to fracture type ${ }^{\text {a }}$ (Danis-Weber classification)

\begin{tabular}{|c|c|c|c|c|}
\hline \multirow[t]{2}{*}{ Reoperations } & \multirow[t]{2}{*}{$N(\%)$} & \multicolumn{3}{|c|}{ Fracture type } \\
\hline & & A-type & B-type & C-type \\
\hline Open debridement and hardware removal & $17(4.5)$ & $4(23.5 \%)$ & $6(35.3 \%)$ & $7(41.2 \%)$ \\
\hline Arthroscopic debridement & $57(15.1)$ & $5(8.8 \%)$ & $24(42.1 \%)$ & $28(49.1 \%)$ \\
\hline Arthrodesis & $8(2.1)$ & 0 & $1(12.5 \%)$ & $7(87.5 \%)$ \\
\hline Total & $82(21.7)$ & $9(11 \%)$ & $31(37.8 \%)$ & $42(51.2 \%)$ \\
\hline
\end{tabular}

${ }^{a}$ Values are expressed as number of reoperations and percentage. 
Brown et $\mathrm{al}^{37}$ reported $32 \%$ of postoperative residual pain related to soft-tissue impingement with overlying plate or screw implants.

Redfern et $\mathrm{al}^{40}$ identified the presence of a neuroma as cause of postoperative residual pain in $15.0 \%$ of their patients. Neuroma was due to transaction of the superficial peroneal nerve during ankle lateral approach. In our experience, no cases of neuroma were found.

Utsugi et $\mathrm{al}^{41}$ described postoperative arthrofibrosis in $73.0 \%$ of the cases during consecutive arthroscopic examinations performed at the time of implant removal. In our study, implant removal and ankle arthroscopy were not performed routinely, but only in case of complications and this explains our smaller incidence (1.9\%).

Srinivasan and Moran ${ }^{42}$ and Leach and Fordyce ${ }^{43}$ described ankle fracture malunion in 5 and $7.9 \%$ of the cases, respectively. These rates are higher than that reported in this study (2.4\%).

In our experience, reoperations were represented by open debridement and hardware removal, arthroscopic debridement, and ankle fusion. Open debridement of all necrotic and fibrous tissue and hardware removal in case of malunions, infections, extra-articular impingement, and implant breakage represented a valid solution to pain relief and joint motion improvement.

Arthroscopic joint debridement is the gold standard to remove loose bodies, osteophytes, and bone spurs causing intra-articular impingement in early posttraumatic ankle $\mathrm{OA}$ (Kellgren-Lawrence 1, 2, or 3 and van Dijk 1 or 2 grade) as well as inflamed synovial tissue, around the joint. This is particularly effective in young and active patients with significant successful rates in terms of pain relief and joint motion. ${ }^{37,41-46}$

Ankle fusion remains the treatment of choice for advanced posttraumatic ankle OA (Kellgren-Lawrence grade 3 or 4 and van Dijk grade 3). The goal of the procedure is to reduce pain by eliminating motion in the osteoarthritic joint. ${ }^{47,48}$ Although arthrodesis is a successful operation in adult or elderly patients, young and active patients do not bear ankle fusion.

We observed impaired wound healing as dehiscence, edge necrosis, and blistering of wound in $3.2 \%$ cases. Also, this prevalence differs from the literature data, which vary from 5.2 to $9.0 \%$. $^{10,42}$

Finally, CRPS had similar incidence to that reported in the literature. $^{49-51}$

Our study has some limitations. This study is not a multicenter or registry study and all data were collected from medical records, surgical procedure, and outpatient control reports.

In conclusion, ankle fracture ORIF represents a satisfying surgical treatment; nevertheless, postoperative complications are not uncommon. Minor complications can be easily managed with medications and repeated outpatient controls. Reoperation is occasionally required to treat major complications. Revision surgery is mandatory to ensure pain relief and function improvement.

\section{Conflict of Interest}

None.

\section{References}

1 Court-Brown CM, Caesar B. Epidemiology of adult fractures: a review. Injury 2006;37(08):691-697

2 Tadros AM, Eid HO, Abu-Zidan FM. Epidemiology of foot injury in a high-income developing country. Injury 2010;41(02):137-140

3 SooHoo NF, Krenek L, Eagan MJ, Gurbani B, Ko CY, Zingmond DS. Complication rates following open reduction and internal fixation of ankle fractures. J Bone Joint Surg Am 2009;91(05):1042-1049

4 Seland K, Cherry N, Beach J. A study of factors influencing return to work after wrist or ankle fractures. Am J Ind Med 2006;49(03): 197-203

5 Asloum Y, Bedin B, Roger T, Charissoux JL, Arnaud JP, Mabit C. Internal fixation of the fibula in ankle fractures: a prospective, randomized and comparative study: plating versus nailing. Orthop Traumatol Surg Res 2014;100(4, Suppl):S255-S259

6 Ovaska M. Complications in ankle fracture surgery. Acta Orthop Suppl 2015;86(358):1-32

7 Kannus P, Palvanen M, Niemi S, Parkkari J, Järvinen M. Increasing number and incidence of low-trauma ankle fractures in elderly people: Finnish statistics during 1970-2000 and projections for the future. Bone 2002;31(03):430-433

8 Schepers T, De Vries MR, Van Lieshout EMM, Van der Elst M. The timing of ankle fracture surgery and the effect on infectious complications; a case series and systematic review of the literature. Int Orthop 2013;37(03):489-494

9 Thangarajah T, Prasad PS, Narayan B. Surgical site infections following open reduction and internal fixation of ankle fractures. Open Orthop J 2009;3(03):56-60

10 Ebraheim NA, Mekhail AO, Gargasz SS. Ankle fractures involving the fibula proximal to the distal tibiofibular syndesmosis. Foot Ankle Int 1997;18(08):513-521

11 Leyes M, Torres R, Guillén P. Complications of open reduction and internal fixation of ankle fractures. Foot Ankle Clin 2003;8(01): 131-147

12 Medicode (Firm). ICD-9-CM: International Classification of Diseases, 9th Revision, Clinical Modification. Salt Lake City, UT: Medicode; 1996

13 Rüedi TP, Buckley RE, Moran CG. AO Principles of Fracture Management. New York: Thieme Medical Publishers; 2007

14 Hunt KJ, Hurwit D. Use of patient-reported outcome measures in foot and ankle research. J Bone Joint Surg Am 2013;95(16):e118

15 Olerud C, Molander H. A scoring scale for symptom evaluation after ankle fracture. Arch Orthop Trauma Surg 1984;103(03):190-194

16 Horan TC, Gaynes RP, Martone WJ, Jarvis WR, Emori TG. CDC definitions of nosocomial surgical site infections, 1992: a modification of CDC definitions of surgical wound infections. Infect Control Hosp Epidemiol 1992;13(10):606-608

17 Weber BG. Die verletzungen des oberen sprunggelenkes. 2nd ed. Berne: Verlag Hans Huber; 1972

18 van Bergen CJ, Gerards RM, Opdam KT, Terra MP, Kerkhoffs GM. Diagnosing, planning and evaluating osteochondral ankle defects with imaging modalities. World J Orthop 2015;6(11):944-953

19 Moon JS, Shim JC, Suh JS, Lee WC. Radiographic predictability of cartilage damage in medial ankle osteoarthritis. Clin Orthop Relat Res 2010;468(08):2188-2197

20 van Dijk CN, Tol JL, Verheyen CC. A prospective study of prognostic factors concerning the outcome of arthroscopic surgery for anterior ankle impingement. Am J Sports Med 1997;25(06): 737-745

21 Kijowski R, Blankenbaker D, Stanton P, Fine J, De Smet A. Arthroscopic validation of radiographic grading scales of osteoarthritis of the tibiofemoral joint. AJR Am J Roentgenol 2006;187(03):794-799

22 Tanaka Y, Takakura Y, Hayashi K, Taniguchi A, Kumai T, Sugimoto K. Low tibial osteotomy for varus-type osteoarthritis of the ankle. J Bone Joint Surg Br 2006;88(07):909-913

23 Witteveen AGH, Hofstad CJ, Kerkhoffs GMMJ. Hyaluronic acid and other conservative treatment options for osteoarthritis of the ankle. Cochrane Database Syst Rev 2015;(10):CD010643 
24 Hendrickx RP, Stufkens SA, de Bruijn EE, Sierevelt IN, van Dijk CN, Kerkhoffs GM. Medium- to long-term outcome of ankle arthrodesis. Foot Ankle Int 2011;32(10):940-947

25 Schepers T, Van Lieshout EM, De Vries MR, Van der Elst M. Increased rates of wound complications with locking plates in distal fibular fractures. Injury 2011;42(10):1125-1129

26 Van Son MA, De Vries J, Roukema JA, Den Oudsten BL. Health status, health-related quality of life, and quality of life following ankle fractures: a systematic review. Injury 2013;44(11): 1391-1402

27 Zalavras CG, Christensen T, Rigopoulos N, Holtom P, Patzakis MJ. Infection following operative treatment of ankle fractures. Clin Orthop Relat Res 2009;467(07):1715-1720

28 Lee MS, Grossman JP. Complications in Foot and Ankle Surgery: Management Strategies. Cham, Switzerland: Springer; 2017

29 Zalavras CG, Christensen T, Rigopoulos N, Holtom P, Patzakis MJ. Infection following operative treatment of ankle fractures. Clin Orthop Relat Res 2009;467(07):1715-1720

30 Viol A, Pradka SP, Baumeister SP, et al. Soft-tissue defects and exposed hardware: a review of indications for soft-tissue reconstruction and hardware preservation. Plast Reconstr Surg 2009; 123(04):1256-1263

31 Backes M, Schep NW, Luitse JS, Goslings JC, Schepers T. The effect of postoperative wound infections on functional outcome following intra-articular calcaneal fractures. Arch Orthop Trauma Surg 2015;135(08):1045-1052

32 Goost H, Wimmer MD, Barg A, Kabir K, Valderrabano V, Burger C. Fractures of the ankle joint: investigation and treatment options. Dtsch Arztebl Int 2014;111(21):377-388

33 Lindsjö U. Operative treatment of ankle fracture-dislocations. A follow-up study of 306/321 consecutive cases. Clin Orthop Relat Res 1985;(199):28-38

34 Lübbeke A, Salvo D, Stern R, Hoffmeyer P, Holzer N, Assal M. Risk factors for post-traumatic osteoarthritis of the ankle: an eighteen year follow-up study. Int Orthop 2012;36(07):1403-1410

35 Fleischer GM, Warncke P. Late results following ankle joint osteosyntheses [in German]. Zentralbl Chir 1989;114(06):381-387

36 Müller J, Plaass U, Willenegger H. Late results after surgically treated malleolar fractures. II [in German]. Helv Chir Acta 1971;38 (03):329-337

37 Brown OL, Dirschl DR, Obremskey WT. Incidence of hardwarerelated pain and its effect on functional outcomes after open reduction and internal fixation of ankle fractures. J Orthop Trauma 2001;15(04):271-274

38 Chaudhary SB, Liporace FA, Gandhi A, Donley BG, Pinzur MS, Lin SS. Complications of ankle fracture in patients with diabetes. J Am Acad Orthop Surg 2008;16(03):159-170

39 Dawe EJ, Jukes CP, Ganesan K, Wee A, Gougoulias N. Ankle arthroscopy to manage sequelae after ankle fractures. Knee Surg Sports Traumatol Arthrosc 2015;23(11):3393-3397

40 Redfern DJ, Sauvé PS, Sakellariou A. Investigation of incidence of superficial peroneal nerve injury following ankle fracture. Foot Ankle Int 2003;24(10):771-774

41 Utsugi K, Sakai H, Hiraoka H, Yashiki M, Mogi H. Intra-articular fibrous tissue formation following ankle fracture: the significance of arthroscopic debridement of fibrous tissue. Arthroscopy 2007; 23(01):89-93

42 Srinivasan CM, Moran CG. Internal fixation of ankle fractures in the very elderly. Injury 2001;32(07):559-563

43 Leach WJ, Fordyce MJ. Audit of ankle fracture fixation in the elderly. J R Coll Surg Edinb 1994;39(02):124-127

44 Lee YS, Chen SW. Lateral fixation of open AO type-B2 ankle fractures: the Knowles pin versus plate. Int Orthop 2009;33(04):1135-1139

45 Miller AG, Margules A, Raikin SM. Risk factors for wound complications after ankle fracture surgery. J Bone Joint Surg Am 2012; 94(22):2047-2052

46 Weatherall JM, Mroczek K, McLaurin T, Ding B, Tejwani N. Posttraumatic ankle arthritis. Bull Hosp Jt Dis (2013) 2013;71(01): 104-112

47 Coester LM, Saltzman CL, Leupold J, Pontarelli W. Long-term results following ankle arthrodesis for post-traumatic arthritis. J Bone Joint Surg Am 2001;83-A(02):219-228

48 Buck P, Morrey BF, Chao EY. The optimum position of arthrodesis of the ankle. A gait study of the knee and ankle. J Bone Joint Surg Am 1987;69(07):1052-1062

49 Beerthuizen A, Stronks DL, Van't Spijker A, et al. Demographic and medical parameters in the development of complex regional pain syndrome type 1 (CRPS1): prospective study on 596 patients with a fracture. Pain 2012;153(06):1187-1192

50 Rewhorn MJ, Leung AH, Gillespie A, Moir JS, Miller R. Incidence of complex regional pain syndrome after foot and ankle surgery. J Foot Ankle Surg 2014;53(03):256-258

51 Reuben SS. Preventing the development of complex regional pain syndrome after surgery. Anesthesiology 2004;101(05):1215-1224 